\title{
Results of Hodoyoshi-3 and -4 Earth Observation Missions
}

\author{
By Yoshihide Aoyanagi, ${ }^{1)}$ Akira IwaSAKI,${ }^{1)}$ Shinichi NAKAsUKA, ${ }^{1)}$ Takuji Ebinuma, ${ }^{2)}$ \\ Shinichi KIMURA ${ }^{3)}$ and Tomohiro NARUMI ${ }^{3)}$ \\ 1) Department of Aeronautics and Astronautics, The University of Tokyo, Tokyo, Japan \\ ${ }^{2)}$ Department of Electronics and Information Engineering, Chubu University, Aichi, Japan \\ ${ }^{3)}$ Department of Electrical Engineering, Tokyo University of Science, Chiba, Japan
}

\begin{abstract}
This work describes in-orbit results of the Earth-observation missions of the microsatellites Hodoyoshi-3 and Hodoyoshi-4. These satellites were successfully launched from Russia by the Dnepr launch vehicle on June 19, 2014. Hodoyoshi-3 has observation devices that consist of a medium-resolution camera and a low-resolution one. Hodoyoshi-4 is equipped with a high-resolution multispectral camera that has a push-broom imaging sensor with a ground sampling distance of $6.3 \mathrm{~m}$. The initial observations by Hodoyoshi-3 and -4 were successfully achieved. All cameras are regularly providing Earth-observation images. Due to limitations with the satellite attitude-determination system, geometric correction of the acquired images is carried out by registration to other satellite images. Hodoyoshi- 3 and -4 can provide various Earth-observation results that consist of low-, medium-, and high-resolution images. These images are useful for disaster monitoring, vegetation monitoring, agriculture, and forest management.
\end{abstract}

Key Words: Earth Observation, Microsatellite, Hodoyoshi Systems Engineering

\section{Introduction}

Recently, many small, micro-, and nano-satellites have been launched into low Earth orbit because microsatellites have the advantages of low cost and short-term development. The practical utilization of microsatellites is gradually increasing. In particular, many organizations are launching and operating high-resolution microsatellites. Their future plans are high-frequency and real-time monitoring by using satellite constellations that consist of a few hundred microsatellites. ${ }^{1)}$

Microsatellites Hodoyoshi-3 and Hodoyoshi-4 were successfully launched from Russia by the Dnepr launch vehicle on June 19, 2014. ${ }^{2)}$ These satellites have multiple missions that consist of Earth observation, store and forward, new space utilization, and demonstration of novel components. For its Earth-observation mission, Hodoyoshi-3 has two optical cameras that consist of a medium-resolution one and a low-resolution one. Hodoyoshi-3 supplies land images over a wide area. Moreover, the medium-resolution camera features three-band multispectral observation including the near-infrared band. Hodoyoshi-4 is equipped with a high-resolution multispectral camera that is a push-broom imaging sensor with a light-weight telescope. The multispectral capability of Hodoyoshi-4 includes four spectral bands in the visible and near-infrared spectral ranges. Hodoyoshi-3 and -4 initial observations have been successfully achieved. They are providing various Earth-observation results that consist of low-, medium-, and high-resolution satellite images. Application of these satellite images to disaster monitoring, vegetation monitoring, agriculture, and forest management is expected.

This paper describes the design of optical cameras and in-orbit results of the Earth-observation missions of Hodoyoshi-3 and -4 .

\section{Overview of Hodoyoshi-3 and -4}

"Hodoyoshi" means reasonably reliable systems engineering to realize low-cost and short-term development by setting appropriate reliability. ${ }^{3)}$ The balance between cost and reliability is a very important issue in creating new space markets. Hodoyoshi-3 and -4 were developed in order to demonstrate Hodoyoshi Systems Engineering. Figure 1 shows an overview of Hodoyoshi-3 and -4. These satellites have solar array paddles that are deployed on orbit. The major specifications are summarized in Table 1. The bus subsystems of the two satellites are essentially the same, with the exception of their propulsion systems. The mass of Hodoyoshi-3 is $56 \mathrm{~kg}$, whereas that of Hodoyoshi- 4 is $64 \mathrm{~kg}$. Each satellite has a three-axis attitude control subsystem that consists of a star tracker, a fiber-optical gyroscope, sun sensors, a geomagnetic sensor, reaction wheels, and magnetic torquers. The attitude control subsystem is a high-precision and high-agility one. The mission data including Earth-observation images are transmitted by X-band with a $10 \mathrm{Mbps}$ downlink rate. The Japanese X-band receiving stations are located in the town of Taiki near Hokkaido, on the JAXA/ISAS Sagamihara campus, and at the Tokai University Space Information Center. We conduct the satellite operation, pass scheduling, mission planning, and data analysis at the satellite operation center of the University of Tokyo.

Hodoyoshi-3 and Hodoyoshi-4 were launched into a sun-synchronous orbit with an altitude of $\sim 630 \mathrm{~km}$ on June 19 , 2014. In the first pass after the orbit injection, the signal of each satellite was acquired in Tokyo, and the solar array paddles were deployed during the same pass. Three-axis stabilized attitude control was realized on July 4, 2014. Two weeks after launch, basic Earth-pointing control was successfully completed. That marked the end of the critical 
stabilized attitude control was realized on July 4, 2014. Two weeks after launch, basic Earth-pointing control was successfully completed. That marked the end of the critical operation phase, at which point the mission operation began.

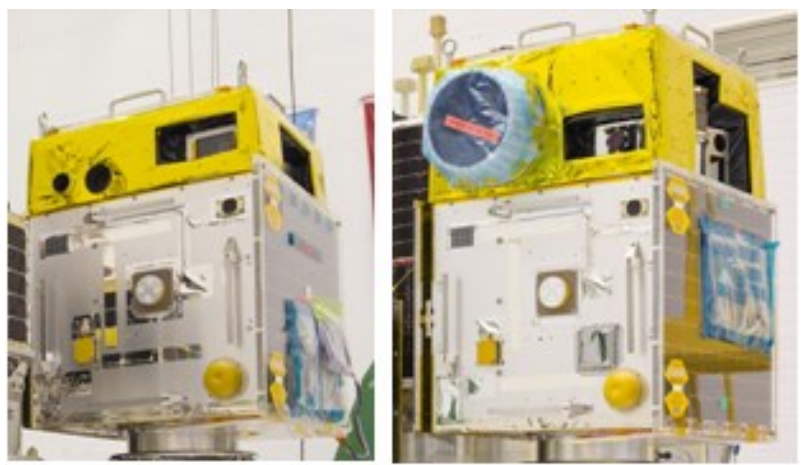

Fig. 1. Overview of Hodoyoshi-3 (left) and -4 (right).

Table 1. Specifications of Hodoyoshi-3 and -4

\begin{tabular}{|c|c|c|}
\hline Item & Hodoyoshi-3 & Hodoyoshi-4 \\
\hline Size & $0.5 \mathrm{~m} \times 0.5 \mathrm{~m} \times 0.7 \mathrm{~m}$ & $0.5 \mathrm{~m} \times 0.6 \mathrm{~m} \times 0.8 \mathrm{~m}$ \\
\hline Weight & $56 \mathrm{~kg}$ & $64 \mathrm{~kg}$ \\
\hline $\begin{array}{l}\text { Attitude } \\
\text { Control }\end{array}$ & \multicolumn{2}{|c|}{ Three-axis stabilization control } \\
\hline Power & \multicolumn{2}{|c|}{$\begin{array}{l}2 \text { solar array paddles, } 5 \text { body-mounted cells } \\
\text { Maximum power generation: } 130 \mathrm{~W} \\
\text { Typical power consumption: } 50 \mathrm{~W} \\
\text { Battery: Li-ion }\end{array}$} \\
\hline $\begin{array}{l}\text { Telemetry } \\
\text { Command }\end{array}$ & \multicolumn{2}{|c|}{$\begin{array}{l}\text { S-band (Telemetry: } 32 / 64 \mathrm{kbps} \text {, Command: } 4 \mathrm{kbps} \text { ) } \\
\text { X-band (Mission data: } 10 \mathrm{Mbps} \text { ) }\end{array}$} \\
\hline $\begin{array}{l}\text { Command } \\
\text { and Data } \\
\text { Handling }\end{array}$ & \multicolumn{2}{|c|}{$\begin{array}{l}\text { On-board computer using SOI-SOC technology } \\
\text { Command Centric Architecture based application }\end{array}$} \\
\hline $\begin{array}{l}\text { Propulsion } \\
\text { system }\end{array}$ & Monopropellant $\mathrm{H}_{2} \mathrm{O}_{2}$ & $\begin{array}{l}\text { Xenon Micro Ion } \\
\text { propulsion }\end{array}$ \\
\hline $\begin{array}{l}\text { Injection } \\
\text { Orbit }\end{array}$ & $\begin{array}{l}\text { Sun-synchronous orbit } \\
\text { LTAN 10:30 } \\
\text { Inclination: } 97.970^{\circ} \\
\text { Altitude: } 612 \mathrm{~km} \times \\
665 \mathrm{~km} \\
\end{array}$ & $\begin{array}{l}\text { Sun-synchronous orbit } \\
\text { LTAN 10:30 } \\
\text { Inclination: } 97.969^{\circ} \\
\text { Altitude: } 612 \mathrm{~km} \times \\
665 \mathrm{~km}\end{array}$ \\
\hline
\end{tabular}

\section{Hodoyoshi-3 Medium-resolution Camera (MCAM) and Low-resolution Camera (LCAM)}

\subsection{Design and characteristics of MCAM and LCAM}

Hodoyoshi-3 has two observation devices consisting of a medium-resolution camera (MCAM) and a low-resolution camera (LCAM). The mass of each compact palm-sized camera is $\sim 200 \mathrm{~g}$. Figure 2 shows an overview of the MCAM and LCAM flight models. All components, including the optical lens and electrical devices, consist of commercial off-the shelf (COTS) components. Although the optical lens is a COTS component, it has silica glass attached in front of it as a radiation blocker. Furthermore, the cameras are equipped with general RGB Bayer CMOS image sensors such as those in digital cameras.

A signal block diagram of the Hodoyoshi-3 mission payload is shown in Figure 3. The two cameras are controlled by an image acquisition system configured with a field-programmable gate array (FPGA). The on-board software uses the LINUX operating system from an open-source resource. The on-board image processing is done with OpenCV, which is an open-source image-processing library. Although it captures raw images during its nominal operation, it can compress them using JPEG. The image acquisition system communicates with the science data-handling unit (SHU) via RS-422 with a bit rate of $1 \mathrm{Mbps}$. Commands from the ground station are received via the SHU.

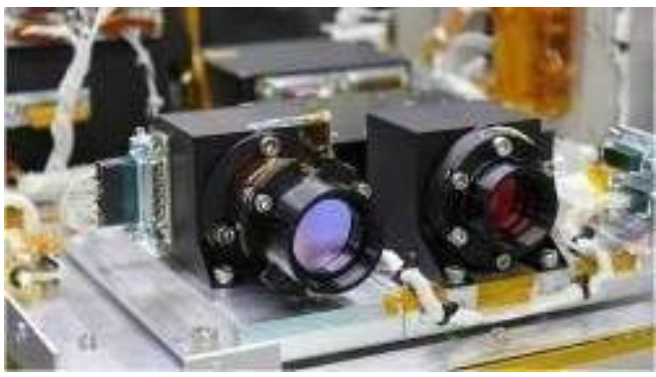

Fig. 2. Overview of medium-resolution camera (left) and low-resolution camera (right)

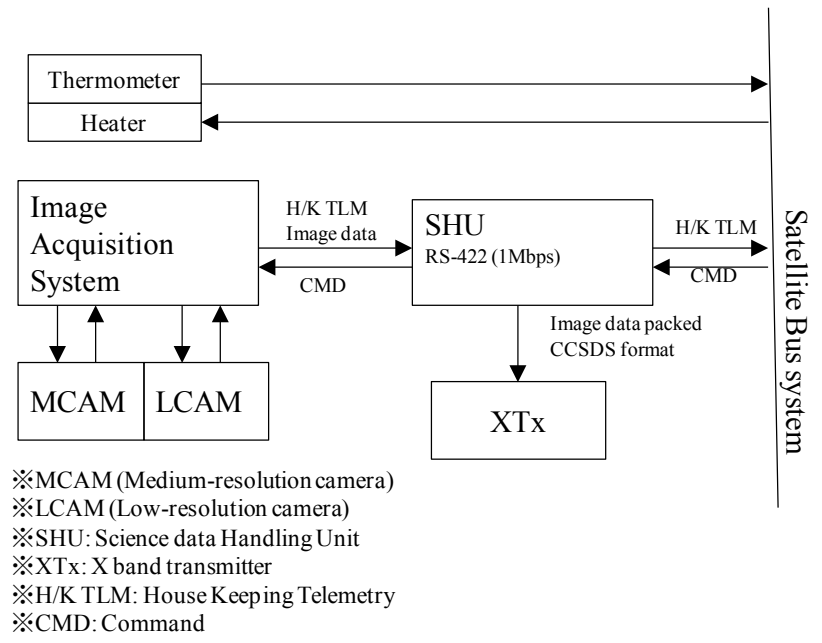

Fig. 3. Signal block diagram of Hodoyoshi-3 mission equipment.

The MCAM and LCAM specifications are summarized in Table 2. The MCAM captures near-infrared, red, and green spectral images with a ground sampling distance (GSD) of $40.3 \mathrm{~m}$ and a swath width of $82.5 \mathrm{~km}$. It features near-infrared observation capability with a general small Bayer-type CMOS image sensor. The near-infrared observation is implemented in a multispectral band-pass filter. Figure 4 shows the principles of infrared, red, and green observation with an RGB Bayer image sensor and a multispectral band-pass filter. The red and green output includes that of the infrared. Hence, Fig. 4(c) shows a graph of the infrared output subtracted from the red and green ones. The correlation of the spectral bands with those of other Earth-observation satellites such as Landsat is not considered because the MCAM and LCAM image sensors have their original color filters attached. Typical vegetation indices are calculated using the near-infrared and red spectral bands. Therefore, the MCAM is expected to be used for vegetation monitoring, such as agriculture, forest, and environmental remote sensing. 
The LCAM has a wide-angle lens with an 8 -mm focal length. It captures RGB color images with a GSD of $252 \mathrm{~m}$ and a swath width of $516 \mathrm{~km}$. It is characterized by very wide-angle view. Therefore, the LCAM is expected to be used for wide-area and rapid observation on a global scale.

Table 2. Specifications of MCAM and LCAM (Hodoyoshi-3).

\begin{tabular}{|l|l|l|}
\hline Item & MCAM & LCAM \\
\hline Image size & \multicolumn{2}{|l|}{$2048 \times 1536$ pixels } \\
\hline GSD @630 km & $40.3 \mathrm{~m} /$ pixel & $252 \mathrm{~m} /$ pixel \\
\hline Area @630 km & $82.5 \mathrm{~km} \times 61.9 \mathrm{~km}$ & $516 \mathrm{~km} \times 387 \mathrm{~km}$ \\
\hline Spectral band & $\begin{array}{l}\text { G: } 550-580 \mathrm{~nm} \\
\text { R: } 650-690 \mathrm{~nm} \\
\text { IR: } 890-930 \mathrm{~nm}\end{array}$ & RGB color \\
\hline Dynamic range & $10 \mathrm{bit}$ & $10 \mathrm{bit}$ \\
\hline Field of View & $7.5^{\circ}$ & $44.5^{\circ}$ \\
\hline Focal length & $50 \mathrm{~mm}$ & $8 \mathrm{~mm}$ \\
\hline Pixel size & $3.2 \mu \mathrm{m}$ & $3.2 \mu \mathrm{m}$ \\
\hline Mass & $250 \mathrm{~g}$ & $190 \mathrm{~g}$ \\
\hline
\end{tabular}

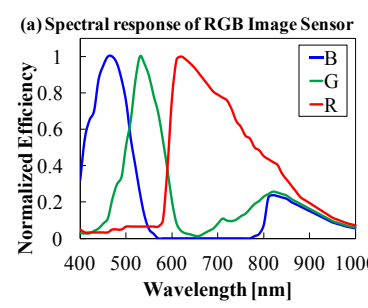

(c) Spectral response of IRG Image Sensor

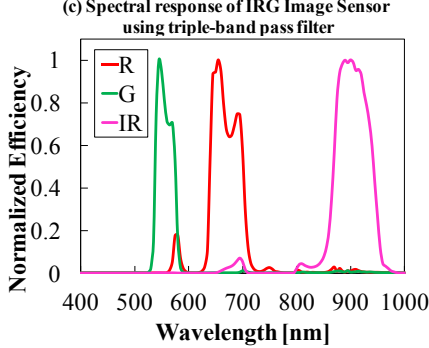

Fig. 4. Near-infrared observation method using multispectral band-pass filter with RGB Bayer CMOS image sensor.

\subsection{Pre-flight imaging performances}

The imaging performances of the MCAM and LCAM were evaluated before flight. Figure 5 shows the line-spread functions (LSFs) of the MCAM and LCAM flight model that was measured by ground tests. Firstly, we measured the modulation transfer function (MTF) by using the collimator with the Siemens star target attached, and then converting the MTF to the LSF. The latter was evaluated after de-mosaic processing. As a result, the MCAM green band achieved a full width at half maximum (FWHM) of $5.9 \mu \mathrm{m}$. This corresponds to two pixels of the image sensor, which has a pixel size of $3.2 \mu \mathrm{m}$. This imaging quality was verified before and after environmental test consisting of vibration, thermal cycle, and vacuum ones. Both the LCAM and MCAM achieved sufficient imaging quality based on the same ground tests.

The LCAM has a very wide-angle view, but this has a large distortion of $-10 \%$. Therefore, the off-axis imaging quality is much less than the on-axis one. Images captured by the LCAM are distortion corrected by off-line image processing.
The MCAM and LCAM were radiometrically calibrated. We used a radiometric calibration system consisting of an integral sphere, a halogen-tungsten lamp, and a spectral radiance meter at the National Institute of Polar Research, Japan. As a result, we calibrated the sensitivity deviation of each pixel and obtained a radiometric correction coefficient for each spectral band.
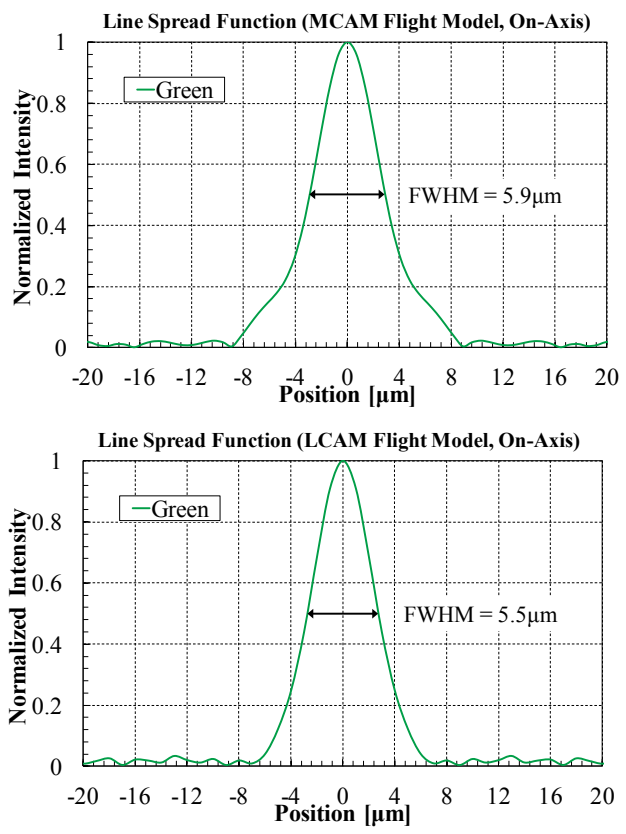

Fig. 5. Line spread functions measured before flight (upper: MCAM; lower: LCAM).

\subsection{Initial operation of Hodoyoshi-3}

The MCAM and LCAM power-on procedures started in parallel with checkout of the attitude parameters on June 24, 2014. The MCAM and LCAM successfully achieved first-light observation on July 5, 2014 (15 days after launch). Figures 6 and 7 are the first-light images captured by the LCAM and MCAM, respectively; both images have been distortion corrected. Due to the absence of a blue spectral band, the MCAM image is displayed as an RGB composite generated from Band 2 (red), Band 3 (infrared), and Band 1 (green). The infrared spectral band was assigned to green in order to highlight green vegetation. Because the thermal control function was not used at first, the temperature of both cameras was $\lesssim 0^{\circ} \mathrm{C}$. The MCAM requires thermal control of $20 \pm 5^{\circ} \mathrm{C}$ to maintain good imaging quality. We therefore consider the MCAM image to have been out of focus at first observation.

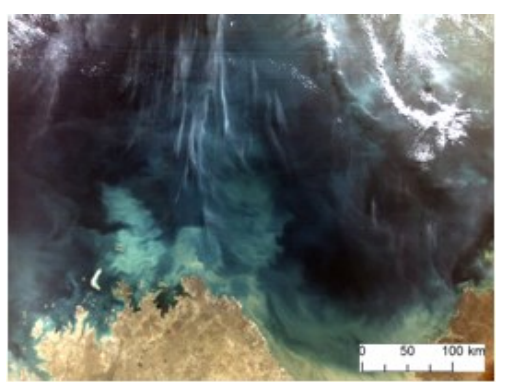

Fig. 6. LCAM first-light image: East Timor (July 5, 2014). 


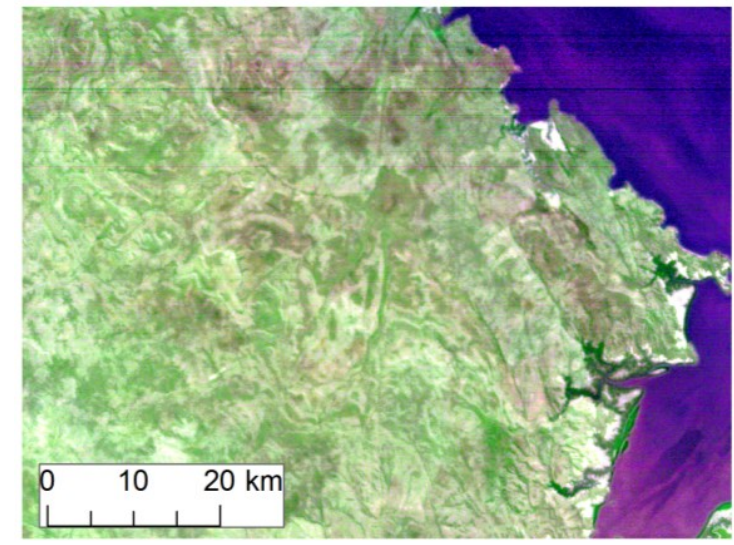

Fig. 7. MCAM first-light image: Australia (July 5, 2014). RGB composite (R-G-B = R-IR-G).

\subsection{Result of Earth observation by Hodoyoshi-3}

Figure 8 shows an image of Japan captured by the LCAM on August 20, 2014. The LCAM provides land images over a very wide area, although we cannot see the detail of individual cities. However, it can acquire highly frequent color images of Japan and other countries because it can capture images from the same position every $1-2 \mathrm{~d}$. We expect LCAM images to be used for rapid observation when a disaster happens on a regional scale.

Figure 9 shows a Tokyo-centered image captured by the MCAM on October 17, 2014. The thermal control function using a heater and a thermometer kept the temperature in the optical lens tube at $20 \pm 5^{\circ} \mathrm{C}$ after the initial operation. Figure 10 shows images taken at $0{ }^{\circ} \mathrm{C}$ and $20^{\circ} \mathrm{C}$ around the lens tube. We could not select identical areas for the comparison, and we did not evaluate in-orbit quantitative performance such as the LSF. However, as a qualitative result, image quality appears improved by using thermal control. Moreover, according to the attitude angle supplied from the altitude control system and the captured image, we estimated that the satellite body axis and the optical axis of the cameras are out of alignment. Therefore, an offset angle is added to the altitude one. Hodoyoshi-3 managed to acquire its target with an accuracy of several degrees as a result of adding this offset angle. Thanks to these operations, the MCAM and LCAM successfully captured target images. This attitude accuracy is not high enough for Earth observation, but we cannot conduct high-precision attitude determination using the star tracker. As an in-orbit result, it is found that the internal parameters of the star-tracker tuning are insufficient. Although we tested the star tracker before launch, a component-parameter tuning problem was found in the internal software of the on-board computer, and hence the star tracker cannot determine the attitude. ${ }^{4)}$

Typical vegetation indices are calculated using the near-infrared (NIR) and red (R) spectral bands. A normalized-difference vegetation index (NDVI) is calculated as NDVI $=(\mathrm{NIR}-\mathrm{R}) /(\mathrm{NIR}+\mathrm{R})$. NDVI image calculated using the MCAM NIR and R bands is shown in Fig. 11. The based image is of a large farm area near Adelaide, Australia on September 16, 2014. As the in-orbit radiometric calibration had not yet been performed, the radiance value of the image was calibrated using the result of the ground tests. The result indicated that vegetation maps could be easily created using the MCAM, although the accuracy of the vegetation index would need further evaluation.

The MCAM and LCAM can take up to 10 consecutive shots at one time. Figure 12 shows the Tohoku region in Japan captured by the MCAM using its continuous-shoot function. The along-track distance is $\sim 220 \mathrm{~km}$. The MCAM and LCAM cannot take high-resolution images unlike Hodoyoshi-4 (GSD: $6.3 \mathrm{~m}$ ), RapidEye (GSD: $6.5 \mathrm{~m}$ ), and other high-resolution satellites. However, MCAM image can cover very wide areas of land, such as Landsat $(170 \mathrm{~km} \times 185 \mathrm{~km})$. The Fukushima area was included in Figure 12, which was used to monitor environmental changes within the Fukushima evacuation zone. ${ }^{5)}$

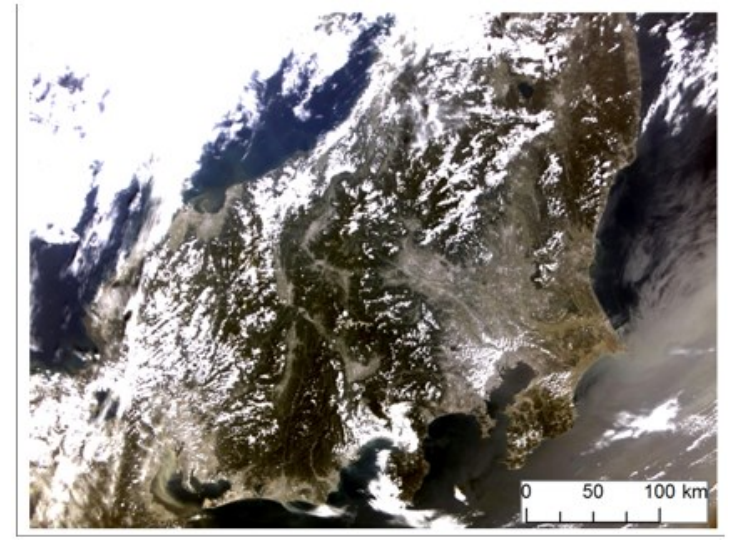

Fig. 8. LCAM image of Japan (August 20, 2014).

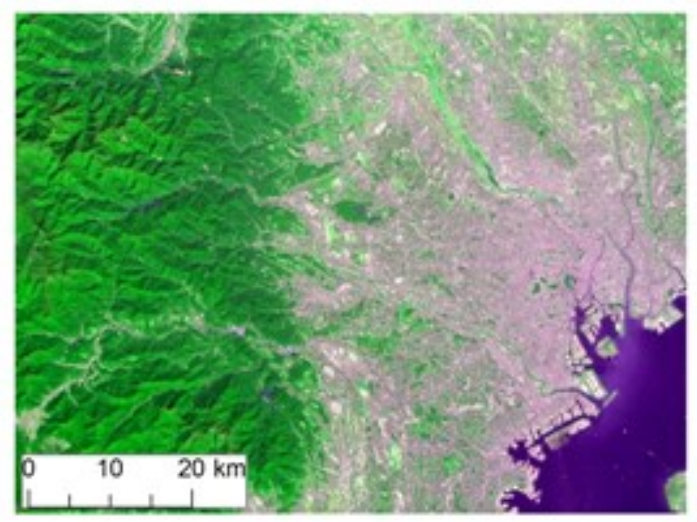

Fig.9. MCAM image of Tokyo, Japan (October 17, 2014). RGB composite (R-G-B = R-IR-G).
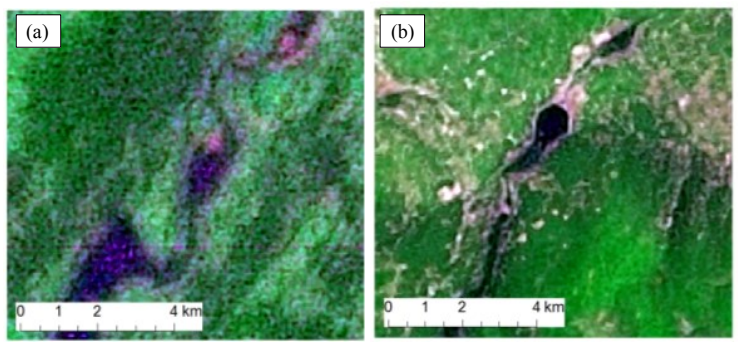

Fig. 10. MCAM focus adjustment using thermal change. Temperature around lens tube: (a) $0{ }^{\circ} \mathrm{C}$, (b) $20^{\circ} \mathrm{C}$. 

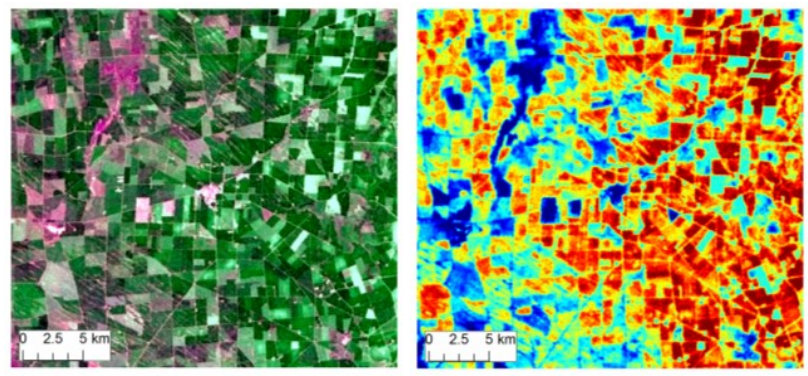

Fig. 11. MCAM RGB composite image (left) and NDVI image (right); Adelaide, Australia (September 16, 2014).

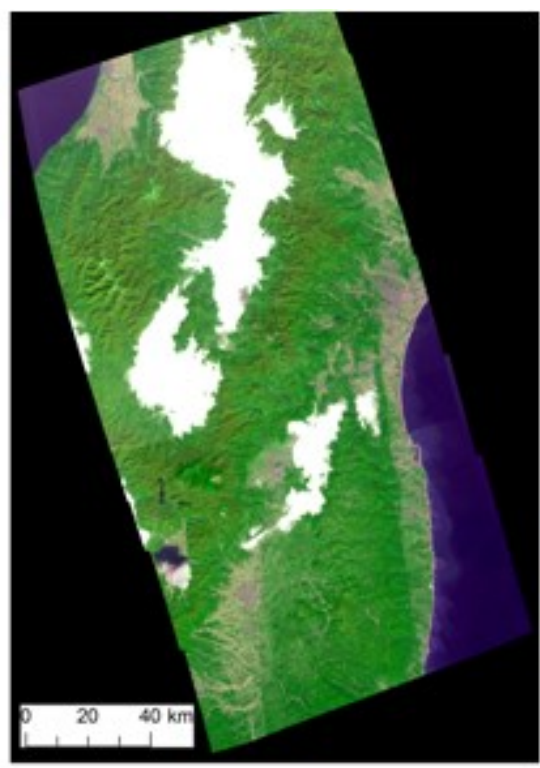

Fig. 12. MCAM image of Tohoku region, Japan (October 17, 2014). RGB composite (R-G-B $=$ R-IR-G).

\section{Hodoyoshi-4 High-resolution Multispectral Camera (HCAM)}

\subsection{Design and characteristics of HCAM}

Hodoyoshi-4 is equipped with the high-resolution multispectral camera (HCAM) shown in Fig. 13. This has a push-broom imaging sensor with a GSD of $6.3 \mathrm{~m}$. The swath width is $25.8 \mathrm{~km}$ at an attitude of $630 \mathrm{~km}$. The multispectral capability includes four bands in the visible and near-infrared spectral ranges. The major parameters are summarized in Table 3.

The optical telescope design is a modified Ritchey-Chrétien type that has a $150-\mathrm{mm}$ aperture with a $1,000-\mathrm{mm}$ focal length. It is known that typical Ritchey-Chrétien telescopes have field curvature. Therefore, the HCAM is equipped with a flattener lens to correct the field curvature and capture good images over a wide field of view. The thermal structure of the optical telescope is lightweight and robust at high temperatures. The main optical tube is made from a carbon-fiber reinforced polymer (CFRP) that has extremely low shrinkage and expansion against heat. However, the thermal heaters are attached to the main optical tube and the base plate. Therefore, the temperature of the telescope is slightly controllable. Also, lightweight glass ceramics are used for the mirror of the optical telescope. A lightweight telescope of $5.2 \mathrm{~kg}$ is realized by using these materials.

Figure 14 shows the HCAM spectral response. It is equipped with two linear CCD KLI-4104 image sensors supplied by Kodak. These consist of three parallel linear arrays, each with 4080 active pixels with a pixel size of $10 \mu \mathrm{m}$ The sensor contains a fourth channel for luminance information, which we did not use. Figure 15 shows the configuration of the linear CCD image-sensor alignment. For visible observation, the HCAM has RGB filters attached to one image sensor. An NIR filter is attached to the other sensor for NIR observation. The HCAM spectral bands were determined with reference to Landsat-7. The spectral response of the NIR covers is $780-890 \mathrm{~nm}$ to avoid the Fraunhofer $(760 \mathrm{~nm})$ and water adsorption $(900 \mathrm{~nm})$ lines.

The HCAM signal block diagram is shown in Fig. 16. SpaceWire is adopted in the communication interface as for image transmission to the SHU, which communicates at 25 Mbps. The image is transmitted to the ground station by $\mathrm{X}$-band at $10 \mathrm{Mbps}$.

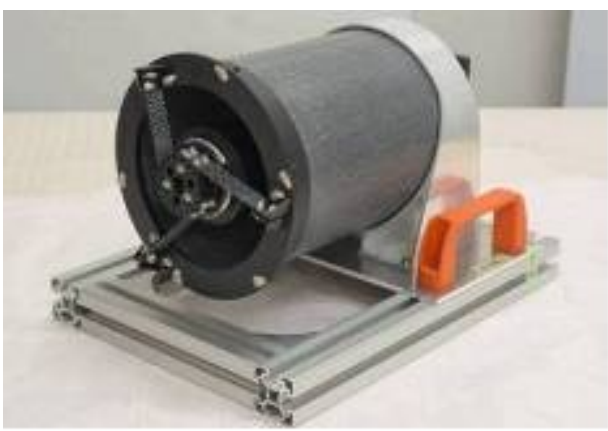

Fig. 13. Overview of HCAM.

Table 3. HCAM major parameters.

\begin{tabular}{|l|l|}
\hline Item & Specification \\
\hline Sensor type & Push broom \\
\hline Focal length & $1000 \mathrm{~mm}$ \\
\hline Aperture size & $150 \mathrm{~mm}$ \\
\hline Field of View & $2.35^{\circ}$ \\
\hline GSD @630 km & $6.3 \mathrm{~m} /$ pixel \\
\hline Swath width @630 km & $25.8 \mathrm{~km}$ \\
\hline Spectral band & B1: 450-520 nm (77) \\
(S/N ratio in case of zenith & B2: 520-600 nm (89) \\
angle 30 , albedo 50\%) & B3: 630-690 nm (62) \\
& B4: 780-890 nm (46) \\
\hline Mass & $5.2 \mathrm{~kg}$ (optical) \\
& $0.65 \mathrm{~kg}$ (electrical) \\
\hline
\end{tabular}

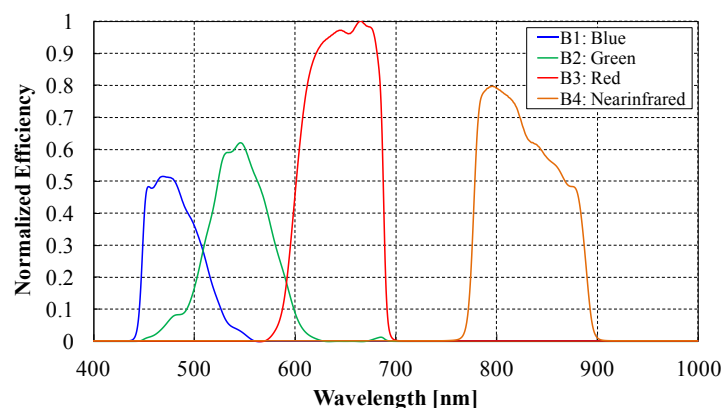

Fig. 14. HCAM spectral responses. 


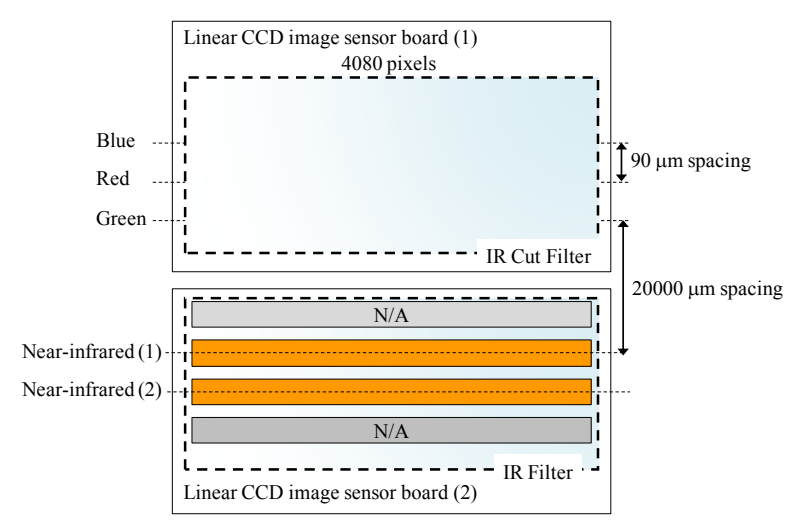

Fig. 15. Alignment diagram of CCD image sensor.

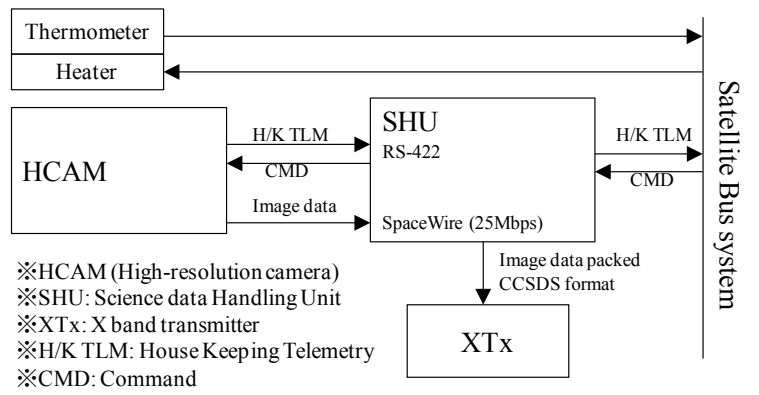

Fig. 16. Signal block diagram of Hodoyoshi-4 mission equipment.

\subsection{Pre-flight evaluation of imaging performance}

The HCAM point-spread function (PSF) was evaluated as the imaging performance by ground tests using a collimator. This PSF was measured using the HCAM CCD linear array with a green laser beam as the light source. Figure 17 shows the PSF of the left-end, center, and right-end positions. The center, left-end, and right-end PSFs are 10.9, 9.9, and $11.2 \mu \mathrm{m}$ $(\sigma)$, respectively. These give $\sim 1$ pixel at $1 \sigma$ over the full field of view. Hence, the HCAM telescope is focused over the full field of view, and we consider that the field curvature is well corrected.
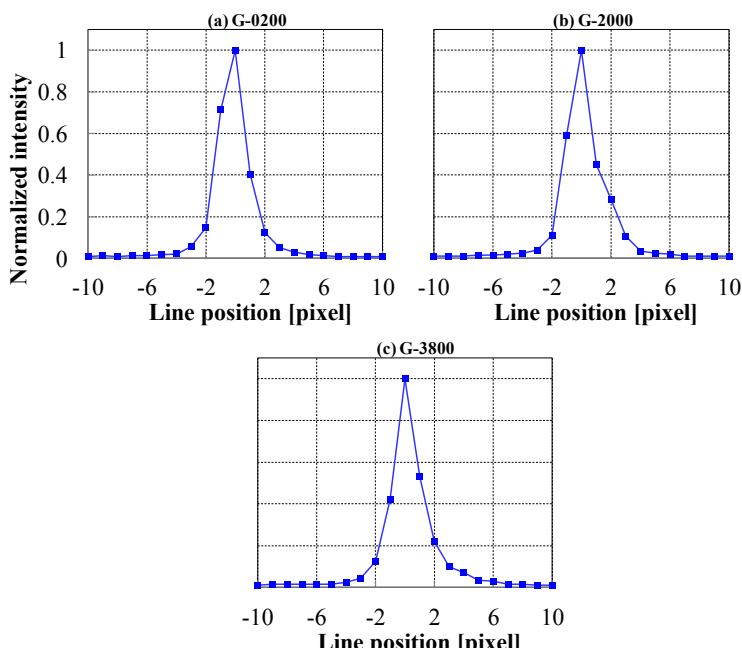

Fig. 17. Point-spread function of HCAM flight model: (a) PSF at left-end position (line position: 200 pixels); (b) PSF at center position (line position: 2,000 pixels); (c) PSF at right-end position (line position: 3,800 pixels).

\subsection{Result of Earth observation by Hodoyoshi-4}

Hodoyoshi-4 realized three-axis-stabilized attitude control on July 4, 2014. For a period of time after that, Hodoyoshi-4 was operated to verify the attitude sensor and other mission devices. The HCAM successfully captured the land image shown in Figure 18 on August 4, 2014. This figure shows picturesque large-scale European farmland. The HCAM captured this image from a greatly inclined state because of the lack of attitude-control-parameters in adjustment. After the initial operation, the offset angles including the alignment error in the targeted pointing angle of Hodoyoshi-4 were adjusted in order to realize high-precision pointing control. However, we could not conduct high-precision attitude determination using the star tracker because the internal parameter tuning was insufficient, as with Hodoyoshi-3. However, Hodoyoshi-4 succeeded in capturing land images with a pointing accuracy of several degrees by coarse attitude-determination system using the gyroscope, sun sensors and geomagnetic sensor.

Figure 18 was captured at an HCAM telescope temperature of $20^{\circ} \mathrm{C}$, but it seems to be defocused compared with the ground-test results. The satellite cannot keep an Earth-facing attitude for a whole day because the solar power generation is insufficient. Also, there are only two temperature measurement and control points in the telescope. Therefore, the temperature of the entire telescope was much lower than we expected. Also, the telescope temperature is uneven. For these reason, we estimated that the HCAM is out of focus even if the temperature is satisfactory $\left(20^{\circ} \mathrm{C}\right)$. Unfortunately, the HCAM is not equipped with a mechanical focus-adjustment mechanism. Therefore, we tried to adjust the focus using thermal expansion. As already described, the thermal structure of the telescope is robust at high temperatures. However, the optical tube for the flattener lens is made of aluminum. Therefore, we tried to raise the temperature of this lens tube using the thermal heater in order to adjust the focus. Figure 19 shows images of an urban area taken by the HCAM at temperatures of $20^{\circ} \mathrm{C}$ and $37^{\circ} \mathrm{C}$ around the optical lens tube. As shown in these images, we can consider the image in the case of $37{ }^{\circ} \mathrm{C}$ to be the better one. We succeeded in focusing the HCAM telescope by increasing the temperature by $\sim 20{ }^{\circ} \mathrm{C}$ above that required. As a qualitative result, the HCAM image quality is improved by using a thermal change.

After this focal adjustment, the HCAM captured high-resolution images, such as the one in Figure 20 of the city of Hiroshima, Japan, that include digital zoom. This image is mainly of an urban area, and we can easily distinguish some of the bridges and rivers. Moreover, we can analyze the detail of the city from this image. The HCAM successfully captures good quality and high-resolution multispectral images, even when compared to existing high-resolution multispectral satellites such as RapidEye (GSD: $6.5 \mathrm{~m}$ ). We consider that the high-resolution multispectral images captured by the HCAM could be used for monitoring environmental changes and disasters in urban areas. 


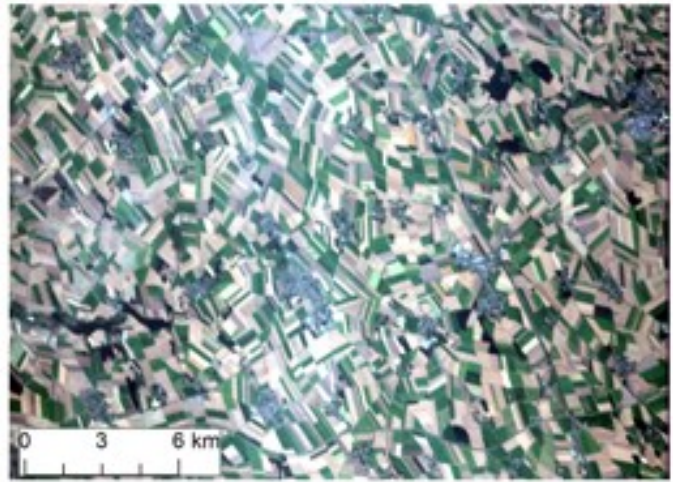

Fig. 18. Initial result of HCAM in agricultural area, France (August 1, 2014).

(a)

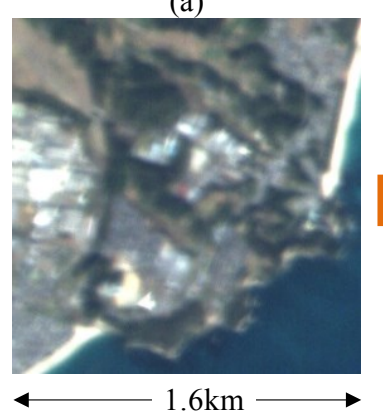

Fig. 19. Focal adjustment result using thermal change: Temperature around lens tube: (a) $20^{\circ} \mathrm{C}$; (b) $37^{\circ} \mathrm{C}$.

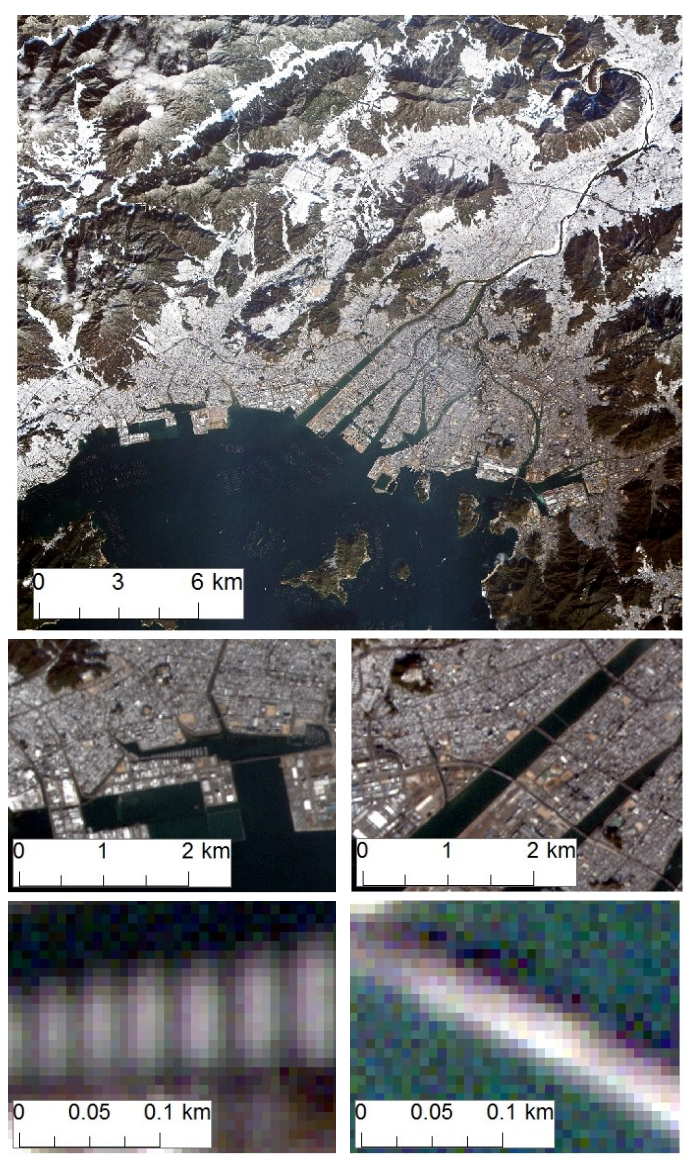

Fig. 20. HCAM image in Hiroshima city, Japan (19, December, 2014)

\subsection{HCAM image processing}

\subsubsection{Radiometric performance}

The HCAM does not have on-board calibration equipment, and its sensitivity was not calibrated before flight. Therefore, the sensitivity characteristic was evaluated using a desert image. Figure 21 shows the limb-darkening characteristics of HCAM Band 3 (red) that was measured over the Kuwait desert. An approximate function was calculated using this data. HCAM images are corrected by this approximate function to reduce the effect of limb darkening.

Table 4 shows the average output of odd- and even-numbered pixels in order to evaluate the stripe noise caused by the dispersion of each CCD image-sensor detector. The difference in output between odd and even is 0.1-1.3. This result shows that the HCAM image sensor does not suffer from stripe noise. Table 4 also gives the $\mathrm{S} / \mathrm{N}$ ratio in each spectral band. The signal is the average value of one line in the image, and the noise is the standard deviation of this signal. For reference, Table 4 includes the spectral radiance of a Landsat-7 image that was captured around the same time. As an evaluated result, the HCAM $\mathrm{S} / \mathrm{N}$ ratio confirms the expected performance.

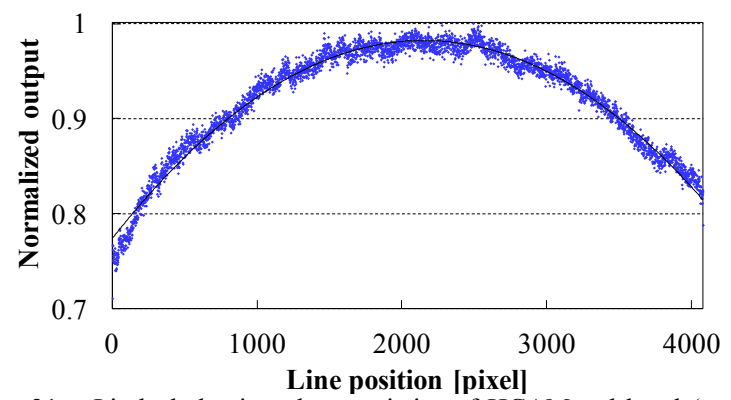

Fig. 21. Limb darkening characteristics of HCAM red band (measured over Kuwait desert, September 14, 2014).

Table 4. Stripe noise and $\mathrm{S} / \mathrm{N}$ ratio after correction for limb darkening.

\begin{tabular}{|l|l|l|l|l|}
\hline Item & B1 & B2 & B3 & B4 \\
\hline Even pixel (ave.) [DN] & 2155.1 & 3239.7 & 3364.9 & 3746.7 \\
\hline Odd pixel (ave.) [DN] & 2154.9 & 3240.3 & 3365.0 & 3745.4 \\
\hline $\begin{array}{l}\text { Difference between odd } \\
\text { and even pixels [DN] }\end{array}$ & -0.26 & 0.55 & 0.15 & -1.30 \\
\hline S/N ratio & 81.2 & 89.5 & 98.3 & 104.4 \\
\hline $\begin{array}{l}\text { Spectral radiance } \\
{\left[\mathrm{W} / \mathrm{m}^{2} / \mathrm{sr} / \mu \mathrm{m}\right](\text { reference }} \\
\text { from Landsat-7) }\end{array}$ & 122 & 140 & 152 & 118 \\
\hline
\end{tabular}

\subsubsection{Geometric correction}

According to the result of an in-orbit experiment on the attitude control system, the altitude information does not meet the requirements of accuracy for precise Earth pointing. Unfortunately, we consider that the satellite altitude information cannot be used for geometric correction. Therefore, geometrically corrected images are created by registration to existing satellite images (i.e., Landsat-8 ones). This processing also includes orthorectification. The following procedures are carried out for the geometric correction of an HCAM image: 
1) Band-to-band registration of the HCAM image;

2) Adjustment of the resolutions of the HCAM and Landsat images;

3) Selection of a ground control point (GCP) from each image;

4) Performance of image-to-image matching by normalized cross-correlation;

5) Attachment of geometric information (e.g., latitude, longitude) to the HCAM image.

Figure 22 shows an HCAM image geometrically corrected by Landsat. HCAM Band 3 was registered using Landsat- 8 Band 4 because the former is spectrally very similar to the latter. Figure 23 presents a comparison of the geometrically corrected HCAM image and the Landsat- 8 one. The registration accuracy between the HCAM and Landsat- 8 images was $<1$ pixel. Moreover, another band including the infrared was registered in the same way. Figure 24 shows composite infrared/red/green and RGB images. Although the parallax between the visible and infrared HCAM images was relatively large, these images are registered with high accuracy, as shown in Figure 24.

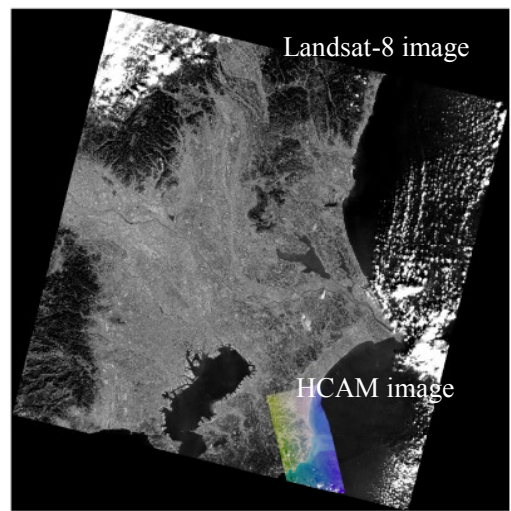

Fig. 22. Geometrically corrected HCAM image based on Landsat-8. HCAM: December 13, 2014; Landsat-8: December 10, 2014.
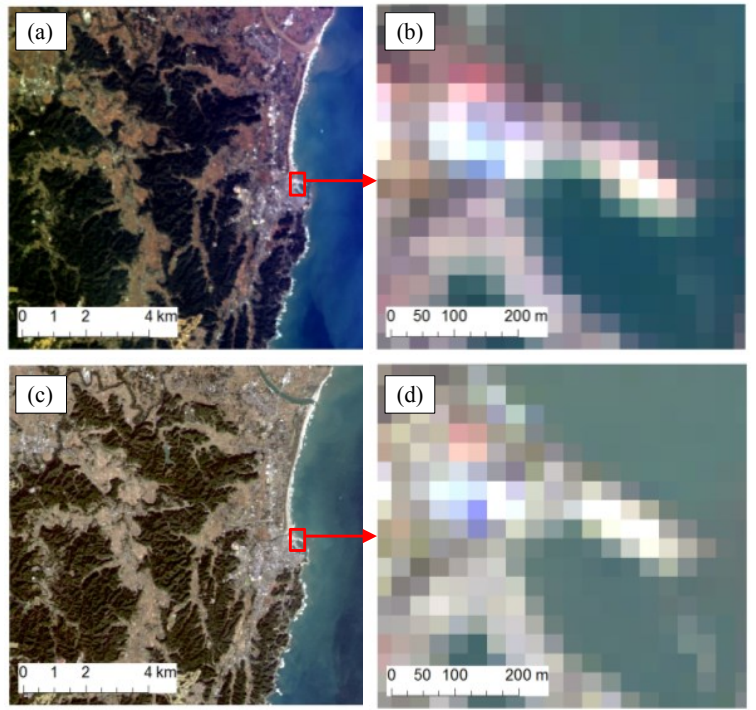

Fig. 23. Comparison of geometrically corrected HCAM images: $(a, b)$ HCAM, (c, d) Landsat-8.
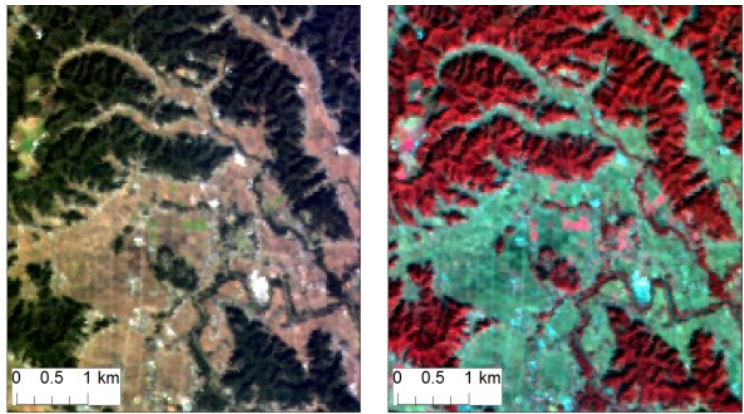

Fig. 24. HCAM image of Chiba, Japan (December 13, 2014): (a) RGB; (b) IRG.

\section{Conclusion}

Hodoyoshi-3 and -4 were successfully launched into a sun-synchronous orbit. They provide regular Earth-observation images at various resolutions. The Hodoyoshi-3 LCAM provides quick land-cover images on a global scale. The MCAM is a very small, palm-sized camera but has near-infrared observation capability. This paper has shown that vegetation maps can be easily created using the MCAM. The Hodoyoshi-4 HCAM successfully captured good quality, high-resolution multispectral images by focal adjustment using thermal control. Geometrically corrected images are produced by registration based on Landsat- 8 ones. Therefore, we can use HCAM and Landsat- 8 images for change detection. We expect Hodoyoshi-3 and -4 to be used in various fields, such as monitoring environmental changes and disasters.

\section{Acknowledgments}

This research is supported by the Cabinet Office, Government of Japan and the Japan Society for the Promotion of Science (JSPS) through the Funding Program for World-Leading Innovative Research and Development on Science and Technology (FIRST Program). This research is partially supported by the Ministry of Education, Culture, Sports, Science and Technology of Japan, Promotion of Space Usage Regulation Consignment Expenses.

\section{References}

1) Kumagai, J.: 5 Earth-imaging Start-ups Coming to a Sky near You, IEEE Spectrum, 51.4, 20-21, 2014

2) Mase, I., Matsui, M., Tsuruda, Y. and Nakasuka, S.: Initial Performance in Orbit of Hodoyoshi-3 and Hodoyoshi-4, 1K10, 58th Space Sciences and Technology Conference, Nagasaki, November, 2014.

3) Nakasuka, S.: From Education to Practical Use of Nano-satellites Japanese University Challenge towards Low Cost Space Utilization -, 8th IAA Symposium on Small Satellite for Earth Observation, Berlin, April, 2011

4) Miyata., K. and Nakasuka. S.: On-Orbit Evaluations of the Three Axis Stabilization Performances of Standard Micro-Satellite Buses, Proceedings of the 30th International Symposium on Space Technology and Science, 2015-f-88p, Kobe, July 2015.

5) Aoyanagi, Y., Iwasaki, A., Nakasuka, S. and Yoshimoto, S.: Environmental Monitoring within Fukushima Evacuation Zone by Multiple satellites including Microsatellites, Proceedings of the 58th Spring Conference of the Remote Sensing Society of Japan, Chiba, June, 2015. 